\title{
7 Kurzfassung: Sterben dürfen im Krankenhaus
}

Hintergrund: Krankenhäuser sind und bleiben perspektivisch der häufigste Sterbeort in Deutschland, und der Tod wird mit der wachsenden Zahl chronisch verlaufender Erkrankungen immer seltener plötzlich eintreten. Sterbebegleitung auf Normal- und Intensivstationen eines Krankenhauses wird damit ein wichtiges Thema für alle beteiligten Akteure und ihre Zusammenarbeit sowie für die medizinische Behandlung und pflegerische Versorgung. Ausgangspunkt der Untersuchung war zudem ein identifizierter Widerspruch zwischen gesundheitspolitischen Überlegungen, öffentlicher Skandalisierung der Behandlung Sterbender und der Behandlungspraxis im Krankenhaus. Trotz der vielfachen wissenschaftlichen Belege für den Nutzen palliativmedizinischer Versorgung im gesamten Behandlungsverlauf besteht eine Forschungslücke hinsichtlich der organisatorischen und sozialen Bedingungen der Integration bzw. der Gründe für ihr Misslingen im deutschen Gesundheitswesen. Die Rolle von Ärzt^innen zeigt sich als zentral bei Behandlungsentscheidungen, auch am Lebensende. Damit erscheinen sie als Schlüsselpersonen, um die Behandlung schwerstkranker und sterbender Patient^innen im Krankenhaus zu untersuchen.

Methode: Die Grounded Theory Methodologie ist Grundlage des explorativen und zirkulären Vorgehens in der Untersuchung. Den organisatorischen Kontext bilden zwei städtische Krankenhäuser mit je einer Palliativstation und einem Palliativkonsildienst. Quantitative und qualitative Kontextanalysen, 30 Leitfadeninterviews mit Ärztinnen und Ärzten aus 13 verschiedenen medizinischen Fachdisziplinen, die auf Normal- und Intensivstationen tätig sind, sowie die Ergebnisse einer Gruppendiskussion mit den interviewten Ärztinnen bilden die Datengrundlage. Die Gruppendiskussion dient zudem als Validierungstrategie, ebenso wie eine forschungsbegleitende Studiengruppe.

Ergebnisse: Im Kontext eines Akutkrankenhauses mit einer ökonomisierten „Durchlaufmedizin“ und routinierten „Arbeit im Akkord“ verunsichern Sterbende oder Langlieger als nicht heilbare Patient`innen den medizinischen Enthusiasmus. Zudem erschüttert die Behandlungspraxis die ärztlichen Ideale von Zeit und Raum für die Sterbebegleitung. Diese verunsicherte, weil uneindeutige, Behandlungssituation wird in der Palliativ-Dimension und in heterogenen Sterbebildern deutlich. Um den zentralen Behandlungsauftrag der Akutbehandlung bzw. Heilung im Krankenhaus aufrechtzuerhalten, lassen sich verschiedene ärztliche Strategien erkennen: eine begriffliche Erweiterung der Akut- und/oder Heilungsorientierung um eine „formal kurative“ Behandlung, eine diffuse, vermeidende oder einseitig auf Heilung ausgerichtete ärztliche Aufklärung von Patient*in und Angehörigen trotz Verschlechterung der Erkrankungssituation, eine Priorisierung von kurativen vor palliativen Patient*innen bei der Aufnahme, eine schnelle Verlegung Schwerstkranker oder Sterbender aus dem eigenen Arbeitsbereich oder aber die paradoxe Nicht-Nutzung eines Palliativ- 
konsildienstes trotz formuliertem Unterstützungsbedarf. Das ärztliche Entscheiden wird auch für Sterbesituationen als zentraler Behandlungsauftrag angesehen. Patient*innenverfügungen werden für die Entscheidungsfindung als wenig hilfreich erlebt, anders als die Einschätzung der Pflegenden, die eine Therapiezieländerung oftmals einleitet. Die Sterbebegleitung und die Unterstützung der nun zunehmend in Erscheinung tretenden Angehörigen, nicht aber Therapieentscheidungen, werden im Aufgabenbereich der Pflegekräfte verortet. Die komplexen Entscheidungssituationen verweisen auf Hierarchien und eine starke Personengebundenheit, d. h. auf individuelle und strukturelle Handlungsspielräume. In der Schlüsselkategorie, dem ,ärztlichen Postulat vom Sterbendürfen im Krankenhaus“, formiert sich eine Theorie der Praxis, welche die widersprüchlichen Forderungen und Begründungen für die gegenwärtige medizinische Behandlung schwerstkranker und sterbender Patient*innen im Krankenhaus aufgreift. Der vorrangig intradisziplinäre und organisationsbezogene Appell der Ärztinnen verweist auf eine diffuse Bewusstheit in der Behandlung Sterbender und auf eine stark hierarchische Organisation des deutschen Gesundheitswesens.

Diskussion: Die vielfache Fehlversorgung Sterbender im Krankenhaus ist, nicht nur im Hinweis auf den Pflegekräftemangel, verbunden mit einer prekären Arbeitssituation. Das Krankenhaus zeigt sich als wichtiger Lernort, dem eine strukturelle und organisatorische Anerkennung der Lernsituation fehlt. Die vorliegenden Befunde fordern ausgehend von der Rekonstruktion der ärztlichen Entscheidungshierarchie bei gleichzeitiger Beauftragung der Pflege als zuständig in der Sterbebegleitung zudem professionstheoretische Überlegungen heraus. Ein interdisziplinäres Missverständnis über das Behandlungsziel Lebensqualität mit seinen Auswirkungen auf die kollegiale und sektorale Zusammenarbeit sowie auf die Ärzt*in-Patientin-Kommunikation stellt sich als deutliche Integrationshürde einer palliativen Perspektive in die Akutbehandlung dar. Forschung und Praxis sind aufgefordert, dies zu berücksichtigen. Das „ärztliche Postulat vom Sterbendürfen im Krankenhaus“ liefert einen theoretischen Beitrag für ein weiter auszuarbeitendes Sterbedispositiv. 